\title{
Dispersión de semillas de Mauritia flexuosa (Arecaceae) por frugívoros terrestres en Laguna Azul, Beni, Bolivia
}

\author{
Geovanna MENDIETA-AGUILAR ${ }^{1,2, \dagger}$, Luis F. PACHECO ${ }^{1,2^{*}}$, Alejandra I. ROLDÁN ${ }^{2}$ \\ ${ }^{1}$ Universidad Mayor de San Andrés, Instituto de Ecología, Campus Universitario, Calle 27 Cota Cota, La Paz, Bolivia ( $+=$ In memoriam) \\ ${ }^{2}$ Centro de Estudios en Biología Teórica y Aplicada (BIOTA), Av. Las Retamas No. 15, entre calles 34 y 35, Cota Cota, La Paz, Bolivia \\ *Autor correspondiente: luispacheco11@yahoo.com
}

\section{RESUMEN}

El acopio esparcido ha recibido singular atención en las últimas décadas, y muchos estudios se han centrado en semillas de palmeras dispersadas por Dasyprocta. Nuestro objetivo fue identificar las especies consumidoras de frutos de Mauritia flexuosa, evaluar la importancia relativa de esas especies y el destino de las semillas dispersadas. Utilizamos experimentos de campo para evaluar el destino de las semillas removidas por frugívoros, en la Reserva de la Biosfera Pilón Lajas, Bolivia. Predijimos que las semillas enterradas por Dasyprocta tendrían menor mortalidad que las no enterradas. Colocamos 6-16 estaciones de frutos, con 15-50 frutos cada una, en cinco periodos de muestreo cubriendo un año. Los principales dispersores de $M$. flexuosa fueron $D$. punctata y Cuniculus paca, que transportan las semillas un máximo de $12.63 \mathrm{~m}$ y $14.1 \mathrm{~m}$, respectivamente. Los dispersores removieron $7.5 \%$ de los frutos de las estaciones. Todas las semillas dispersadas en cuatro de los muestreos fueron depredadas o se pudrieron; solamente el $0.5 \%$ de las semillas dispersadas en febrero germinaron. De las semillas colocadas sobre y debajo del suelo en época seca y húmeda, solamente sobrevivieron aquellas enterradas en la época húmeda; el resto fueron depredadas o se pudrieron. Ninguna de las 84 semillas removidas por D. punctata fue enterrada, lo cual difiere de casi todos los reportes sobre el comportamiento de Dasyprocta. Discutimos este resultado en relación al posible efecto de la humedad del suelo y el tamaño de las semillas, y postulamos que la elevada abundancia del recurso haría innecesario acopiar semillas bajo suelo.

PALABRAS ClAVE: Scatterhoarding, Dasyprocta, Cuniculus, palmeras, Pilón Lajas

\section{Seed dispersal of Mauritia flexuosa (Arecaceae) by terretrial frugivores in Laguna Azul, Beni, Bolivia}

\section{ABSTRACT}

Scatterhoarding has received special attention for the last couple of decades, and several studies focused on palms whose seeds are dispersed by Dasyprocta. Our objective was to know the species that consume fruits of Mauritia flexuosa, to evaluate the relative importance of consumer species, and also to assess the fate of dispersed seeds. Using field experiments we evaluated the fate of seeds removed by frugivores at the Pilón Lajas Biosphere Reserve, Bolivia. We predicted that seeds buried by Dasyprocta would present lower mortality that those left unburied. We placed 6-16 fruit stations, with 15-50 fruits each, in five sampling periods along a year. The main seed dispersers of $M$. flexuosa were D. punctata and Cuniculus paca, which transported seeds a maximum of $12.63 \mathrm{~m}$, and 14.1, respectively. Dispersers removed $7.5 \%$ of the fruits from the stations. All seeds removed during four out of the five sampling periods were either predated, or rotted; only $0.5 \%$ of the seeds dispersed in February germinated. From the total of seeds we buried in the dry and wet season only survived those buried in the wet season, the remaining were predated or rotted off. None of the 84 seeds removed by D. punctata were buried, which differs from all known reports on dispersal behavior of Dasyprocta. We discuss these findings in relation to the possible effects of soil humidity, and seed size and advance the hypothesis that the high abundance of the resource makes it unnecessary to scatterhoard seeds underground.

KEYWORDS: Scatterhoarding, Dasyprocta, Cuniculus, palms, Pilón Lajas 


\section{INTRODUCCIÓN}

Estudiar el proceso de dispersión de semillas a nivel local es fundamental para entender la estructuración de las comunidades vegetales (Wang y Smith 2002; Howe y Miriti 2004). Las palmas neotropicales producen frutos de semilla grande, abundantes en el bosque y muy atractivos para los frugívoros, especialmente ungulados, roedores y aves (Bodmer 1991; Andreazzi et al. 2009). Algunos mamíferos consumen los frutos y defecan las semillas (Fragoso 1994; Silvius 1999; Quiroga y Roldán 2001), otros llevan las semillas a escondites, donde su supervivencia dependerá de no ser depredadas (Smythe 1970; Tuck-Haugaasen et al. 2012). En este último grupo están roedores como Sciurus, Tamiasciurus, Proechymis, Heteromys, Myoprocta, Dasyprocta, Peromyscus y Reithrodontomys (Bradford y Smith 1977; Alho 1982; Donatti et al. 2009).

Dasyprocta dispersa y entierra las semillas hasta $60 \mathrm{~m}$ desde los parentales (Tuck-Haugaasen et al. 2012); aunque alcanza distancias extremas de $>200 \mathrm{~m}$ (Hirsch et al. 2012a). Este comportamiento de acopio esparcido o "scatterhoarding" (Morris 1962) cumple un rol importante en la dispersión de las semillas (Alho 1982; Jansen et al. 2004; Jorge y Howe 2009). Jansen et al. (2012) reportan que un $14 \%$ de las semillas enterradas por D. punctata sobreviven un año; y las semillas enterradas suelen germinar más rápido que las que no se entierran (Forget y Milleron 1991). Dasyprocta dispersa varios géneros de palmeras: Acrocomia, Astrocaryum, Attalea, Mauritia, Oenocarpus, Phytelephas y Syagrus (Silvius y Fragoso 2003; Pires y Galetti 2012; Emsens et al. 2013). A su vez, frutos y semillas de Mauritia flexuosa están en la dieta de muchos frugívoros, como Tapirus terrestris, Tayasu pecari, Pecari tajacu, Mazama americana, primates, aves e incluso peces (Bodmer 1991; Allen 1997; Holm et al. 2008).

En nuestro sitio de estudio, M. flexuosa (palma real) forma extensos palmares en áreas estacionalmente inundadas. Suponíamos que, siendo D. punctata (jochi colorado) uno de los consumidores (M. Huallata, Poblador local, Com. Pers. 1999), la dispersión de palma real incluiría el acopio esparcido. Suponiendo que éste beneficia a la planta, predijimos que las semillas enterradas tendrían mayor probabilidad de sobrevivencia y germinación que aquellas no enterradas. Adicionalmente, evaluamos si las semillas eran dispersadas diferencialmente hacia áreas permanentemente o temporalmente inundadas, que a su vez albergan poblaciones más y menos densas de $M$. flexuosa, respectivamente (G. Mendieta, datos no publ.). Discutimos estos resultados en relación a la hipótesis de que Dasyprocta acopia más semillas en lugares con menor densidad, donde la probabilidad de establecimiento es mayor (Hirsch et al. 2012b). De forma posterior a nuestro trabajo, se evidenció que el comportamiento de Dasyprocta puede cambiar con la disponibilidad de recursos (Visser et al. 2011; Emsens et al.
2013); y este trabajo tiene especial relevancia en el contexto del modelo de Gálvez et al. (2009), que predice menor densidad de acopios por Dasyprocta en sitios con mayor densidad del recurso. Nuestro objetivo fue identificar las especies que consumen frutos de palma real, evaluar la proporción removida por especie y el destino de las semillas.

\section{MATERIALES Y MÉTODOS}

\section{Área de estudio}

Las observaciones se realizaron alrededor de Laguna Azul, al NO de la Reserva de la Biosfera y Tierra Comunitaria de Origen (RB-TCO) Pilón Lajas, a -377 m snm (140 55’ 45” S y 640 21' 51" W; Figura 1). La vegetación corresponde a Bosque Amazónico Preandino, con temperaturas promedio anuales entre $24-27^{\circ} \mathrm{C}$ y precipitaciones entre 1500 y 2400 $\mathrm{mm}$ anuales (Ibisch et al. 2003), concentrados principalmente entre noviembre y marzo. Los habitantes de la RB-TCO Pilón Lajas son de la etnia T'simane, quienes viven de la agricultura no extensiva, caza y recolección de frutos y otros productos del bosque; lo cual les permite un conocimiento detallado de la historia natural local, incluyendo la fenología de las especies más conspicuas (como M. flexuosa).

Laguna Azul está circundada por un bosque dominado por palma real. En época seca se distinguen dos cinturones de vegetación en el palmar. El cinturón interno (adyacente a la laguna) fuertemente dominado por palma real, cuyos individuos tienen parte del estípite permanentemente bajo el agua y están asociados a vegetación palustre y especies como Nymphea sp., Lemna sp., macrófitas y helechos. El cinturón externo incluye especies que no están en el interno, como Bactris gasipaes, Euterpe precatoria, Astrocaryum murumuru, Ficus sp., Hura crepitans, Annona sp., Rheedia sp. En el cinturón externo, algunos individuos de palma real están en suelo estacionalmente inundado, mientras que otros están en suelo firme. Durante la estación lluviosa el agua cubre entre 10 y $50 \mathrm{~cm}$ de los estípites de las palmas más cercanas al cinturón interno. Las observaciones se realizaron en el palmar, considerando la división en cinturones externo e interno, en función a su potencial importancia para la germinación y establecimiento de $M$. flexuosa. Las densidades de palmas en los anillos interno y externo son de 65 y 55 palmas adultas por hectárea (DS=0.05; G. Mendieta, datos no publ.).

La palma real crece principalmente en suelos ácidos, inundados temporal o permanentemente, donde alcanza alturas de 15 a $30 \mathrm{~m}$ (Moraes 1989). El fruto es una drupa de 5-7 cm de longitud y 4-5 cm de diámetro, con mesocarpio suave, agridulce y de alto valor nutricional (Lopes et al. 1980). La única semilla constituye un $40 \%$ del peso y volumen del fruto; un racimo pesa unos $40 \mathrm{~kg}$. Las semillas son depredadas por varias familias de Coleoptera (Bruchidae, Curculionidae, 


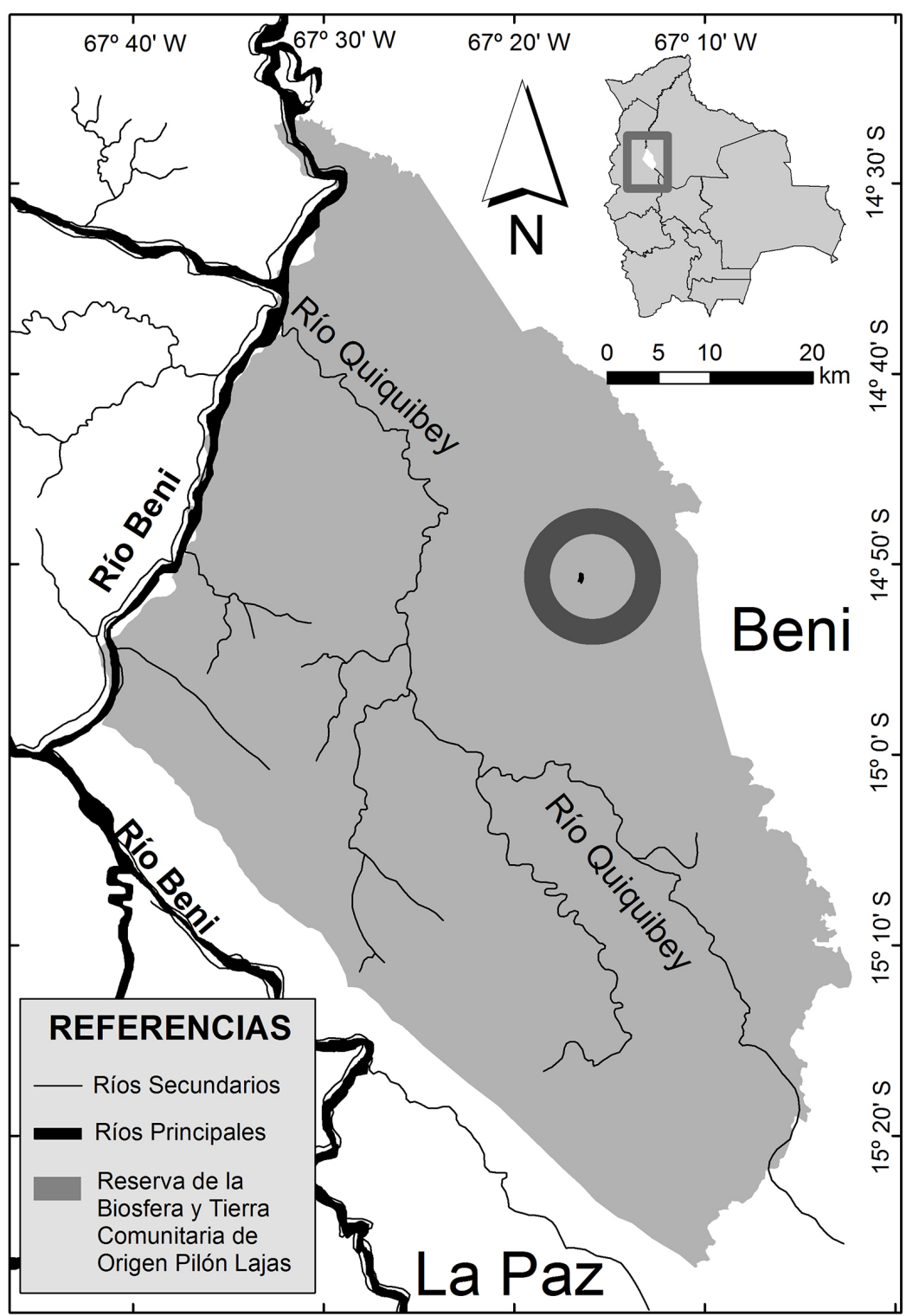

Figura 1. Sitio de estudio, indicado por un círculo, en la Reserva de la Biosfera y Tierra Comunitaria de Origen Pilón Lajas (área sombreada).

Scolytidae) y posiblemente de Hemiptera (Lygaeidae y Pyrrhocoridae; Johanson 2009). La floración ocurre entre septiembre y febrero, y la fructificación entre marzo y octubre (Moraes 1993). En la RB-TCO Pilón Lajas la etapa de fructificación ocurre principalmente entre agosto y diciembre, pero se encuentran frutos todo el año. La germinación es lenta e hipogea y se desarrolla en el agua, con tiempos de germinación entre 82-120 días (Braun 1988).

\section{Dispersión de semillas}

Diseñamos un experimento de campo para identificar los frugívoros terrestres de palma real, estimar la remoción de frutos y evaluar el destino de las semillas dispersadas. Para ello se colocaron estaciones de frutos separadas entre sí por aproximadamente $300 \mathrm{~m}$, rodeando la laguna y suponiendo que cada estación estaría en el área de acción de un jochi colorado (Silvius y Fragoso 2003, Aliaga-Rossel et al. 2008). Las estaciones fueron colocadas a $2 \mathrm{~m}$ desde una palma adulta, para simular la oferta natural de frutos y aproximadamente en el límite observable entre los cinturones externo e interno del palmar; siempre en el mismo lugar que la primera vez o cerca $(<5 \mathrm{~m})$. El número de estaciones y de frutos/estación varió entre muestreos, debido a la distinta disponibilidad de frutos (Tabla 1). Cada estación consistió en un grupo de frutos 
Tabla 1. Número de estaciones y número de frutos por estación colocados en cada ocasión durante el estudio de dispersión de semillas de Mauritia flexuosa por frugívoros terrestres en Bolivia. ES=estación seca; EH=estación húmeda, $\mathrm{T}=$ transición

\begin{tabular}{lccc}
\hline Muestreo & Estaciones & Frutos / estación & Días de muestreo \\
\hline Agosto -1999 (ES) & 6 & 15 & 5 \\
Noviembre -1999 (T) & 14 & 25 & 6 \\
Diciembre -1999 (EH) & 16 & 25 & 7 \\
Febrero -2000 (EH) & 16 & 25 & 7 \\
Agosto -2000* (ES) & 16 & 50 & 7 \\
\hline
\end{tabular}

*Para este muestreo se colocaron dos grupos de 25 frutos cada uno en las estaciones. Uno de los grupos no tenía la pieza de metal, para poder comparar remoción con y sin esa pieza.

(recolectados maduros del árbol) colocados sobre el suelo, alrededor del cual se limpió un área de $1 \mathrm{~m}^{2}$ para registrar huellas de los animales visitantes (Simonetti y Huareco 1999). Las estaciones se instalaron en tres ocasiones (muestreos) en época seca (agosto y noviembre de 1999 y agosto de 2000) y dos en época húmeda (diciembre de 1999 y febrero de 2000).

Los frutos fueron marcados con una pieza de metal $(6 \times 2$ $\mathrm{mm}$ ) firmemente adherida sobre el endocarpio por presión, a excepción de aquellos colocados en agosto de 1999 y la mitad de los colocados en agosto de 2000 (ver abajo). Este procedimiento permitió hallar los frutos removidos con un detector de metales (marca Fisher, modelo 1225x, California, USA). Para evaluar un posible efecto del metal en la remoción, en agosto de 2000 se colocaron dos grupos de 25 frutos en cada estación, uno al lado del otro. Los frutos de un grupo fueron marcados con piezas metálicas, dejando el otro como control. La remoción de frutos marcados fue ligeramente mayor $(2.25 \%)$ que la de frutos control (0.5\%), lo cual sugiere que el marcado de frutos no afecta su remoción. No detectamos pérdida de las piezas de metal, ni ataques por hongos.

\section{Identificación de frugívoros terrestres}

En la mañana de cada día de muestreo (Tabla 1), se revisaron e identificaron las huellas con ayuda de cazadores T'simane, diferenciando las marcas dentales sobre las semillas. Diferenciamos entre visitas con y sin remoción de frutos. Las observaciones se presentan como la proporción promedio de estaciones con visitas por día, para cada especie y cada ocasión de muestreo. La proporción de estaciones con visitas fue correlacionada con la proporción de estaciones donde ocurrieron visitas con remoción, por especie.

\section{Remoción de frutos}

Para cada periodo de muestreo, registramos el número de frutos removidos, desaparecidos e intactos por estación. Frutos removidos fueron aquellos consumidos y depositados por los frugívoros en la misma estación o fuera de ella; aquellos que no se encontraron se contabilizaron como desaparecidos.
Los frutos no removidos y sin indicios de consumo fueron considerados intactos. Los frutos removidos fueron localizados con el detector de metales, rastreando un radio de $30 \mathrm{~m}$ desde cada estación. El instrumento detecta metales hasta $5 \mathrm{~cm}$ bajo suelo y $D$. punctata, el único consumidor que enterraría las semillas, las deposita hasta $4 \mathrm{~cm}$ bajo suelo (Fragoso 1994). La remoción de frutos se midió al final de cada muestreo como la proporción total de remoción por frugívoro y por estación.

Como medida cualitativa de disponibilidad de frutos se estimó la abundancia relativa de frutos sobre el suelo durante los cinco muestreos y tres visitas de campo adicionales en junio de 1999, abril y junio de 2000 para relacionarla con la proporción de remoción. La abundancia se categorizó como alta, media o baja, según la experiencia (> 30 años) del guía local. Para evaluar si la remoción de frutos estaba asociada a una mayor frecuencia de visitas de los frugívoros se utilizó una correlación de Spearman entre el porcentaje de estaciones con visita y el porcentaje total de frutos removidos por día, para cada periodo de muestreo.

\section{Dirección y distancia de deposición de los frutos removidos}

Se midió la distancia desde la estación de frutos hasta el lugar donde se los encontraba, anotando si las semillas eran llevadas hacia el cinturón interno o hacia el externo. Adicionalmente, se comparó la distancia de deposición de frutos entre D. punctata y Cuniculus paca (jochi pintado), el otro mamífero importante en la remoción de frutos en el sitio de estudio, considerando las distancias de deposición entre las áreas inundable y no inundable para cada especie, mediante una prueba de Kruskal Wallis. No utilizamos ANDEVA de dos vías, pues se contravenían severamente los supuestos de normalidad y homoscedasticidad (Zar 1999).

\section{Sobrevivencia de semillas removidas}

La sobrevivencia de las semillas de frutos removidos se evaluó marcando individualmente con estacas etiquetadas los sitios donde se las localizaba. Evaluamos la proporción de semillas germinadas, desaparecidas e intactas por muestreo, para el total de frutos removidos por cada especie. Las semillas intactas son aquellas sin daño por depredación de invertebrados. Las semillas marcadas se revisaron de 1-3 veces en función a la sobrevivencia; hasta un máximo de 180 días desde su remoción.

\section{Germinación y sobrevivencia de semillas bajo y sobre suelo}

Para evaluar si la germinación y sobrevivencia de las semillas difería entre aquellas depositadas sobre y bajo el suelo, hicimos dos pruebas de germinación, una en época húmeda (febrero-2000) y otra en época seca (junio-2000); consistentes en colocar una semilla a $5 \mathrm{~cm}$ bajo el suelo y junto 
a esta una semilla sobre el suelo, en 30 sitios distribuidos al azar alrededor del cinturón externo del palmar. Las semillas fueron recolectadas de frutos maduros hallados en suelo. Se removió el pericarpio y se seleccionaron cuidadosamente semillas sin indicios de depredación por invertebrados. Considerando que las semillas tardan 65-120 días en germinar (Braun 1988), registramos germinación y sobrevivencia de las semillas germinadas después de 69, 120 y 194 días en época húmeda y después de 74 días en época seca. Cuantificamos la proporción de semillas intactas, germinadas, desaparecidas, depredadas y podridas, por sitio (debajo y sobre el suelo), y por época (húmeda y seca). La frecuencia de semillas con dańo (depredadas por invertebrados y podridas) debajo y sobre el suelo, se comparó por época con una Prueba de $x^{2}$ de contingencia (Siegel y Castellan 1995).

\section{RESULTADOS}

\section{Consumidores de Mauritia flexuosa}

Un total de 2040 frutos fueron colocados en las estaciones en los cinco periodos de muestreo (Tabla 1), 7.40\% fue removido por frugívoros y $0.83 \%$ desaparecieron (fueron removidos, pero no encontrados). El mayor porcentaje de remoción corresponde al jochi colorado $55.6 \%(\mathrm{n}=84)$ y jochi pintado $41.7 \%(n=63)$ del total removido. El mayor número de estaciones con visitas y remoción de frutos por ambas especies se registró en época seca, agosto de 1999 (Figura 2). Mazama americana y T. terrestris removieron sólo un fruto cada uno; y roedores no identificados removieron dos frutos. Las remociones por ungulados y roedores se excluyeron de posteriores evaluaciones. Sin embargo, cabe notar que se hallaron heces de T. terrestris con semillas de $M$. flexuosa aparentemente en buen estado y viables.

La tasa de visitas de los frugívoros a las estaciones y la proporción total de remoción de frutos se correlacionaron positivamente para todas las fechas de muestreo: agosto de $1999\left(\mathrm{r}_{\mathrm{s}}=0.885 ; \mathrm{p}<0.001\right)$, noviembre $\left(\mathrm{r}_{\mathrm{s}}=0.883 ; \mathrm{p}<\right.$ $0.001)$, diciembre $\left(r_{s}=0.74 ; p<0.001\right)$, febrero $\left(r_{s}=0.77 ; p\right.$ $<0.001)$ y agosto de $2000\left(\mathrm{r}_{\mathrm{s}}=0.59 ; \mathrm{p}<0.001\right)$; lo cual sugiere que la actividad de los frugívoros cerca de las estaciones estaba influenciada por la disponibilidad de los frutos en el sitio de estudio. Esto coincide con el hecho que la mayor cantidad de frutos removidos se dio en agosto de 1999, cuando, según la experiencia de nuestro guía de campo, había mayor disponibilidad en el ambiente; y la menor en agosto de 2000.

\section{Dirección y distancia de deposición de los frutos removidos}

La distancia máxima a la que las semillas fueron dispersadas por jochi colorado y jochi pintado fue de $12.63 \mathrm{~m}$ y 14.04

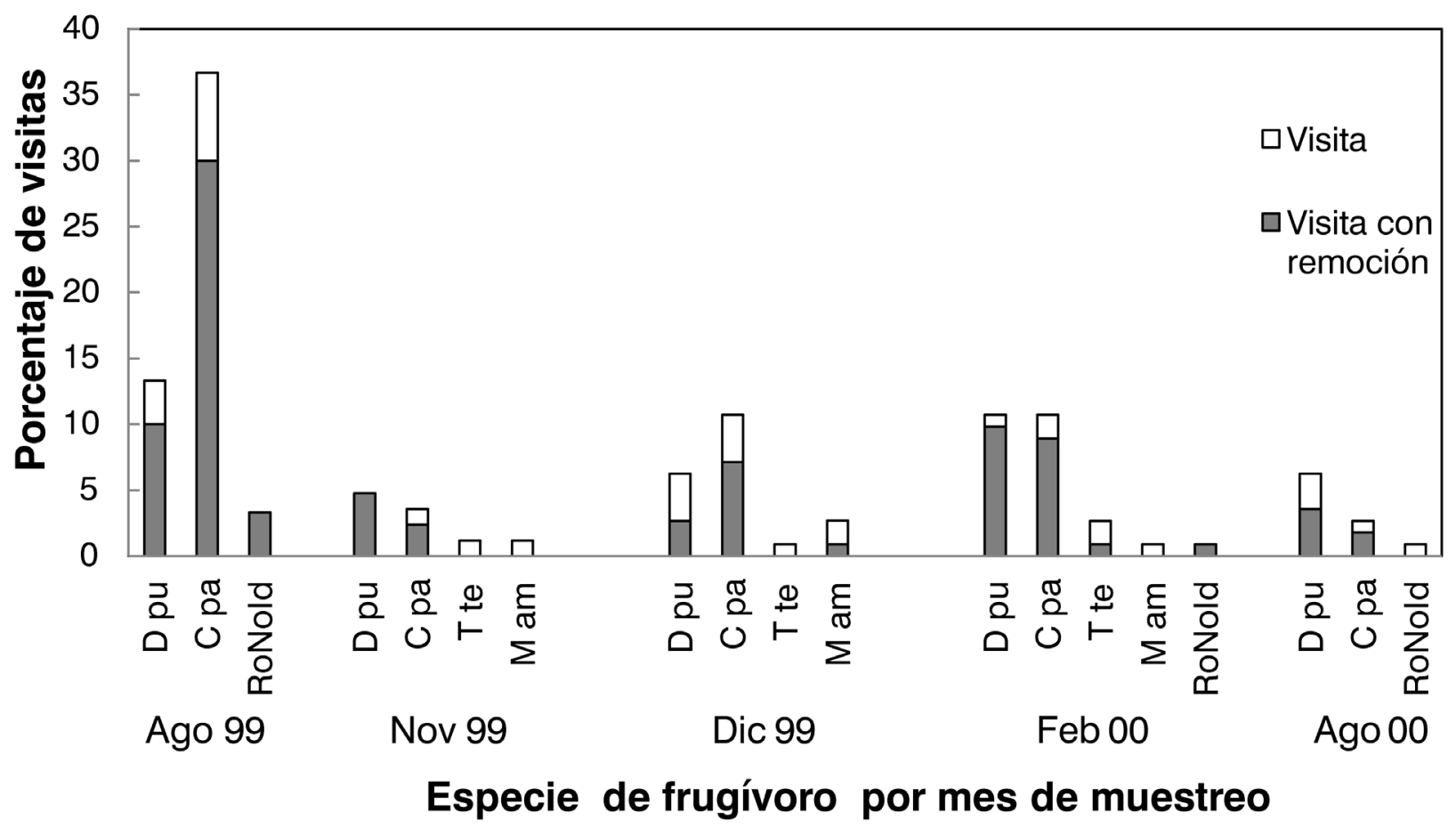

Figura 2. Visitas totales y visitas con remoción de $M$. flexuosa registradas en las estaciones de frutos en cada ocasión de muestreo. $D$ pu=Dasyprocta punctata, $C$ $\mathrm{pa}=$ Cuniculus paca, $\mathrm{T}$ te $=$ Tapirus terrestris, $\mathrm{M}$ am $=$ Mazama americana, RoNold $=$ Roedores no identificados, Ago $=$ agosto $1999 ;$ Ago $00=$ agosto 2000 
$\mathrm{m}$, respectivamente, ambas en la época húmeda (febrero). Generalmente, el jochi colorado dejaba las semillas a $<1 \mathrm{~m}$ de la estación, mientras que el jochi pintado dejaba la mayoría entre 1 y $5 \mathrm{~m}$. Las semillas que fueron alejadas a mayor distancia por jochi colorado y jochi pintado quedaron en área inundable (cinturón interno).

La distancia de deposición de las semillas por jochi colorado considerando todas las fechas de muestreo $(\bar{y}=1.92$ $\mathrm{m}$; rango: $1.00-12.63 ; \mathrm{N}=67$ ) fue menor que por jochi pintado $(\bar{y}=3.27 \mathrm{~m}$; rango $1.00-14.04 \mathrm{~m} ; \mathrm{N}=41 ; \mathrm{z}=$ -4.41, $\mathrm{p}<0.001)$. La distancia de deposición de semillas por jochi colorado y jochi pintado difirió entre las áreas inundable y no inundable (Kruskall Wallis $\mathrm{H}[\mathrm{g} . \mathrm{l} .=3, \mathrm{~N}=108]=25.42$, $\mathrm{P}<0.01)$. La menor distancia de deposición fue por jochi colorado $(\bar{y}=1.13 \mathrm{~m} ; 1.0-3.5 \mathrm{~m} ; \mathrm{N}=34)$ hacia el área inundable, aunque no difirió significativamente de la distancia para esta misma especie hacia el área inundable $(\bar{y}=2.73 \mathrm{~m}$; mediana $=1$, rango $1.00-12.63 \mathrm{~m} ; \mathrm{N}=33$ ); pero sí difirió de las distancias de deposición por jochi pintado hacia el área inundable $(\bar{y}=3.77 \mathrm{~m}$; mediana $=1.79$, rango $1-14.04 \mathrm{~m}$; $\mathrm{N}=10)$ y no inundable $(\bar{y}=3.11 \mathrm{~m}$, mediana $=2.5$, rango $1-12 \mathrm{~m} ; \mathrm{N}=31$; Figura 3).

\section{Sobrevivencia de las semillas removidas}

En general, el jochi colorado removió más frutos que el jochi pintado y las otras especies en todas las ocasiones de muestreo. La sobrevivencia de las semillas difirió entre muestreos. La proporción de semillas depredadas fue mayor que la de semillas desaparecidas y germinadas en cada muestreo (Figura 4). Todas las semillas de los frutos removidos por jochi colorado y jochi pintado en noviembre y diciembre fueron depredadas. Entretanto, dos semillas $(0.5 \%)$ y una semilla $(0.5 \%)$ del total removido en febrero por jochi colorado y jochi pintado, respectivamente germinaron y alcanzaron $5 \mathrm{~cm}$ de altura a los seis meses. No registramos ataque por hongos.

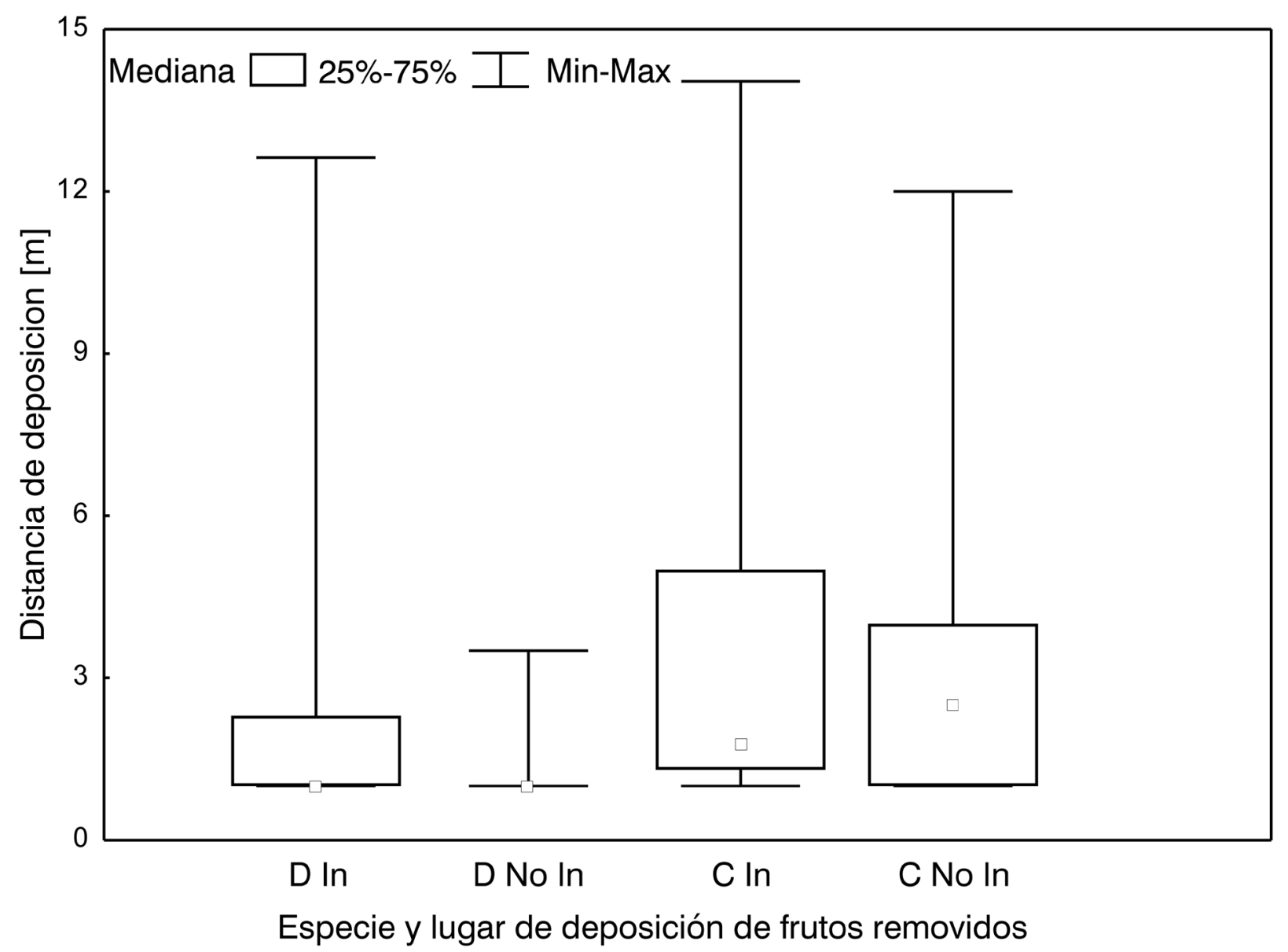

Figura 3. Distancia (en m) y lugar de deposición de frutos removidos por Dasyprocta punctata y Cuniculus paca. D In, D No In (Dasyprocta hacia el área inundable y no inundable, respectivamente); C In, C No In (Cuniculus hacia el área inundable y no inundable, respectivamente). 


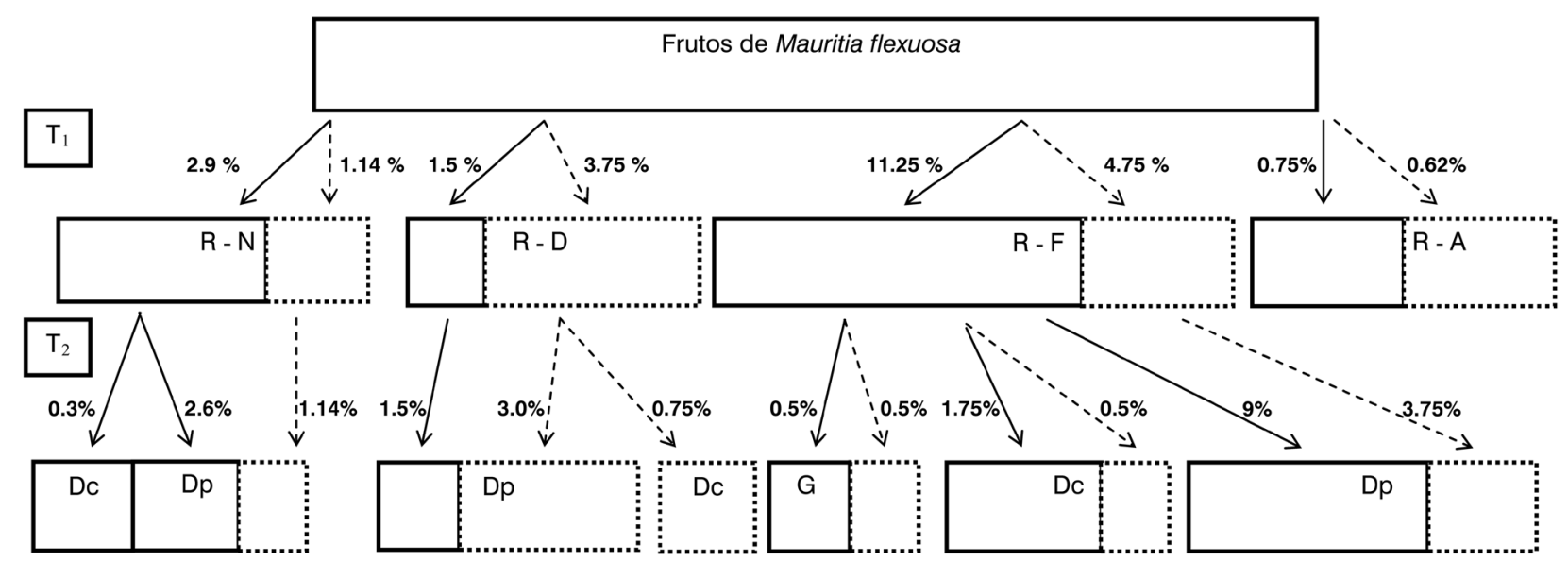

Figura 4. Destino de los frutos de M. flexuosa removidos en el palmar de Laguna Azul. $\mathrm{T}_{1}$ Porcentaje de remoción por $D$. punctata (líneas contínuas) y $C$. paca (líneas punteadas). $T_{2}$ Porcentaje de semillas germinadas $(G)$, depredadas (Dp) y desaparecidas (Dc) luego de ser removidas por $D$. punctata (borde continuo) y C. paca (borde punteado). En T1, el mayor tamaño de los bloques indica mayor consumo de frutos, y en T2 mayor depredación o sobrevivencia de las semillas. $R=$ frutos removidos, $N=$ noviembre, $D=$ diciembre, $F=$ febrero, $A=$ agosto.

Las semillas depositadas en el área inundable por ambos frugívoros fueron las únicas que sobrevivieron y germinaron. Estas semillas fueron cubiertas por agua en la época húmeda. Las semillas desaparecidas fueron aquellas llevadas por el agua, pero se consideran potencialmente viables.

\section{Germinación y sobrevivencia de semillas sobre y bajo el suelo}

El porcentaje de semillas depredadas, tanto aquellas dejadas sobre suelo, como aquellas colocadas debajo el suelo en la primera evaluación, fue menor en la época húmeda (sobre suelo: $6.67 \%$ y debajo suelo: $20 \%$ ), que en la época seca (sobre suelo: $73.3 \%$; debajo suelo: $56.7 \%$ ) para ambos sitios. En tanto que, el porcentaje de semillas podridas difirió entre fechas de muestreo y sitios (sobre y bajo suelo, Figura 5). Sólo quedaron semillas intactas en la época húmeda, en la cual no registramos semillas podridas sobre el suelo. En la época seca la mayoría de las semillas fueron depredadas. La posición de las semillas (sobre o debajo el suelo) tuvo un efecto significativo sólo en la época húmeda, en que la proporción de semillas podridas y depredadas difirió según esa posición $\left(x^{2}\right.$ $=4.1 ;$ g.l. $=1 ; \mathrm{P}<0.05$, Figura 5). Este efecto no fue evidente en la época seca $\left(x^{2}=2.2 ;\right.$ g.l. $\left.=1 ; \mathrm{P}>0.10\right)$. El porcentaje de semillas podridas en la segunda evaluación, fue mayor que el porcentaje de semillas depredadas, tanto debajo como sobre el suelo (debajo suelo: $70 \%$, sobre suelo: $33.33 \%$ ) y (debajo suelo: $20 \%$, sobre suelo: $26.7 \%$ ), respectivamente.

\section{DISCUSIÓN}

Los consumidores terrestres de los frutos de M. flexuosa en el palmar de Laguna Azul fueron D. punctata, C. paca, T. terrestris, $M$. americana y roedores pequeños. Estas especies también consumen frutos de $M$. flexuosa en otros sitios; además de Eira barbara, Chrysocyon brachiurus, T. tajacu, T. pecari, Geochelone sp., edentados y marsupiales (Allen 1997, Holm et al. 2008, Rojas-Robles et al. 2012), que no registramos en este estudio. Esta diferencia podría explicarse simplemente porque nosotros muestreamos un solo sitio (Allen 1997 muestreó cuatro). También es posible que la cacería asociada a extracción maderera cerca a Laguna Azul haya diezmado o ahuyentado a Tayassu pecari, que es una especie sensible a la cacería y actividad humana (Fragoso 1997; Roldán y Simonetti 2001). Las especies más importantes en nuestro estudio por la proporción de frutos removidos fueron D. punctata y C. paca, coincidentemente con Allen (1997), quien registró a $D$. fuliginosa como el principal consumidor de $M$. flexuosa y a $C$. paca como el tercero en importancia.

Es posible que especies como D. punctata y $C$. paca tuvieran mayores probabilidades de encontrar las estaciones de frutos dentro de sus ámbitos de hogar (2-8 ha, Silvius y Fragoso 2003; Aliaga-Rossel et al. 2008), que las especies con ámbitos de hogar más grandes, como T. terrestris (Bodmer 1991; Fragoso 1994; Allen 1997). Silvius y Fragoso (2003) reportan que Dasyprocta puede depender de pocos árboles por varios meses. Adicionalmente, como ya se indicó, la cacería sobre especies de gran tamaño podría haber reducido la abundancia de T. pecari y $T$. terrestris, resultando en una reducción de la remoción de frutos por esas especies.

Dasyprocta es conocido por su hábito de enterrar su alimento para ser luego recuperado y consumido (Smythe 1970, 1978; Kiltie 1981; Forget y Milleron 1991). Específicamente, Peres et al. (1997) observaron que las 


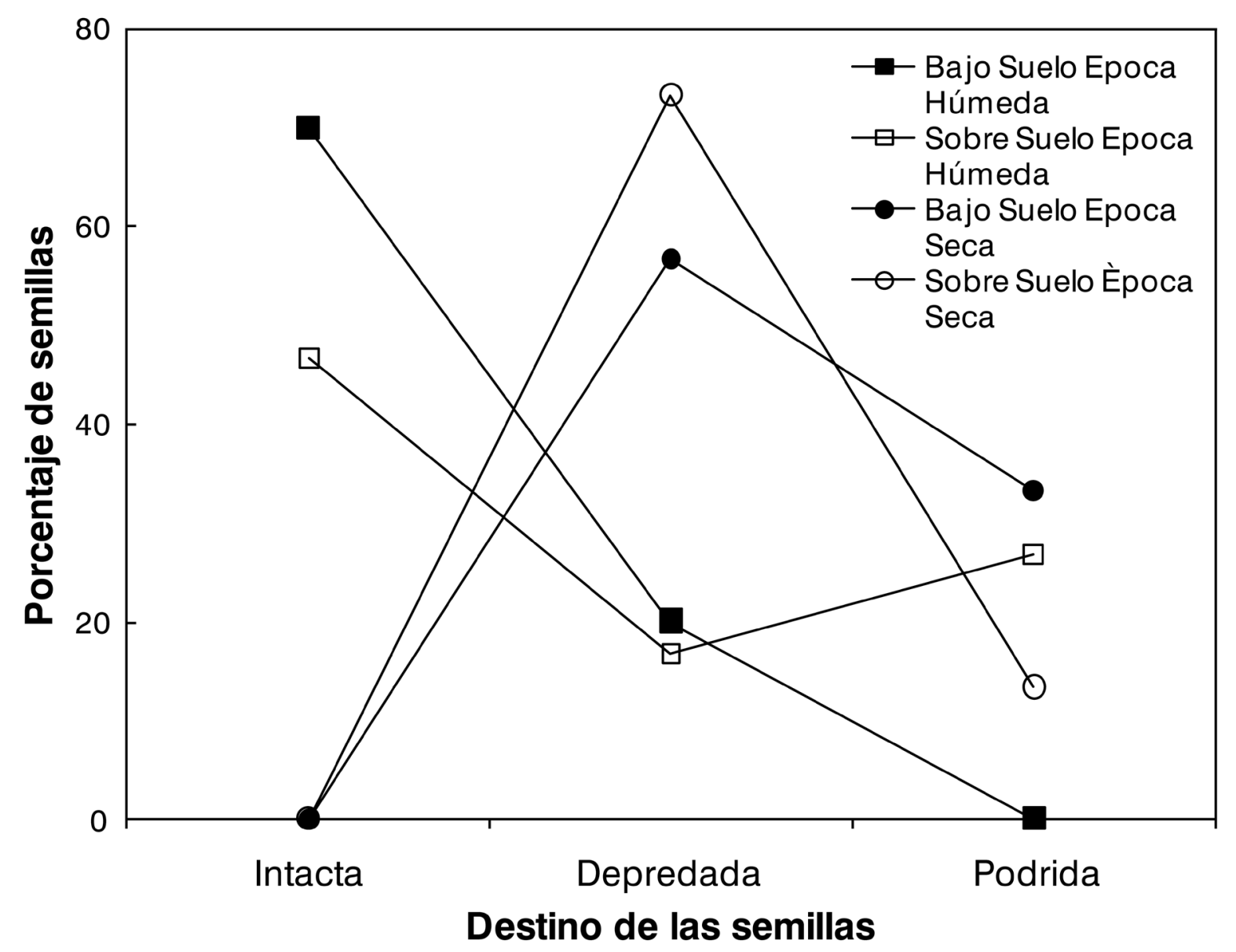

Figura 5. Sobrevivencia de semillas de M. flexuosa colocadas bajo el suelo y sobre el suelo en estaciones seca y húmeda. Los valores corresponden al porcentaje de semillas en cada situación entre los 60 a 70 días después de iniciada la prueba.

semillas de Bertholletia excelsa son enterradas por $D$. leporina en Brasil, Smythe et al. (1982) encontraron que D. fuliginosa entierra semillas de Astrocaryum sp., Dypteryx y Spondias en Isla Barro Colorado; Forget y Milleron (1991) para las semillas de Virola nobilis y las semillas de Astrocaryum aculeatissimum son enterradas por D. Leporina (Pires y Galetti 2012). Sin embargo, no encontramos evidencias que apoyen ese comportamiento para el caso de $D$. punctata y las semillas de $M$. flexuosa en la RB Pilón Lajas. Nuestros resultados de tasas de germinación sobre y bajo el suelo podrían dar luces sobre esta aparente contradicción. Si bien encontramos que la germinación de semillas de palma real es mayor para aquellas ubicadas bajo el suelo, que las que quedan sobre el suelo, tal como lo reportan Forget y Milleron (1991) para las semillas de V. nobilis dispersadas por D. punctata en la Isla de Barro Colorado, y Soares y Ribeiro (1995) para semillas de H. oblongifolia dispersadas por D. agouti en la Reserva do Mocambo en Brasil, la proporción de semillas germinadas bajo el suelo fue 5-8 veces menor en el presente estudio con respecto a lo registrado por Forget y Milleron (1991) y Soares y Ribeiro (1995), respectivamente. Probablemente esta diferencia es causada por la excesiva humedad del suelo en Laguna Azul, que ocasionaría mayor cantidad de semillas podridas. Es decir, las semillas enterradas serían más susceptibles a pudrirse, que aquellas sobre el suelo; por lo cual enterrar las semillas no sería una estrategia adecuada para Dasyprocta, pues luego hallaría la mayoría en mal estado. Esta hipótesis requiere ponerse a prueba, para verificar si Dasyprocta puede cambiar de estrategia según la humedad del suelo y si este cambio obedece a un tipo de plasticidad o es adaptativo para algunas poblaciones. Otra posibilidad es que las semillas no sean enterradas por ser muy pequeñas, ya que las semillas de masa menor a $0.9 \mathrm{~g}$ (i.e. Euterpe edulis) no serían enterradas por Dasyprocta (Galetti et al. 2010); sin embargo, esto no aplica a las semillas de Mauritia, que tienen masas mucho mayores. También se ha observado 
que Dasyprocta prefiere las semillas depredadas por brúquidos sobre aquellas que no lo están (Silvius y Fragoso 2002), debido posiblemente a que los endocarpios con brúquidos son más fáciles de abrir y que las larvas serían una buena fuente de proteínas y lípidos (Fragoso 1997; Silvius y Fragoso 2002). Esta preferencia apoyaría también la hipótesis sobre la estrategia de no enterrar las semillas, pues el hacerlo podría disminuir la probabilidad de depredación postdipsersión por brúquidos, tal como ocurre en las semillas de Attalea phalerata (Ríos y Pacheco 2006), y hacer las semillas menos atractivas por la ausencia de la larva. Finalmente, es posible que la disponibilidad de semillas sea tan alta que enterrarlas no es necesario, pues no habría escasez del recurso. Jorge y Howe (2009) reportan diferencias en el comportamiento de Dasyprocta y Myoprocta para enterrar semillas de Astrocaryum aculueatum, según el tamaño del fragmento de bosque, indicando ya un potencial de plasticidad en ese comportamiento. Gálvez et al. (2009) hallaron una correlación negativa entre la densidad de acopios de semillas (catches) y su disponibilidad en Barro Colorado. Si esta relación se cumpliera hasta el extremo, podría esperarse que la densidad de acopios sea nula, si la disponibilidad del recurso es muy alta en nuestro sitio de estudio; que en el caso de Mauritia flexuosa alcanza densidades entre 55 y 65 adultos ha ${ }^{-1}$. La puesta a prueba de esta hipótesis requiere de un experimento de remoción de semillas en un sitio de alta disponibilidad (como el nuestro), donde Dasyprocta debería retomar el hábito de enterrar semillas, si el comportamiento es plástico, como sugieren Jorge y Howe (2009). Por otro lado, en un lugar con menor disponibilidad de semillas podrían adicionarse grandes cantidades de semillas de alguna especie que es enterrada, de manera que se desencadene el comportamiento de no acopiarlas. En todo caso, será útil considerar el posible efecto de la densidad de Dasyprocta en los sitios, pues se ha visto que esta afecta el comportamiento de enterrar las semillas (Donatti et al. 2009).

Si bien la remoción de frutos de las estaciones por $T$. terrestris no fue importante, el hecho de haber hallado heces de tapir con semillas de $M$. flexuosa enteras y aparentemente sanas es importante. Dado que la materia fecal parece disminuir la depredación por brúquidos en otras especies de palmeras (Silvius 1999; Fragoso y Huffman 2000; Ríos y Pacheco 2006), T. terrestris podría tener un rol crucial en Laguna Azul. Los resultados de algunos estudios describen a T. terrestris como ineficientes dispersores de semillas por defecar las semillas en bajíos que estacionalmente se inundan, lugares probablemente inseguros para la sobrevivencia de A. phalerata (Quiroga y Roldán 2001) y A. maripa (Fragoso 1994). En Laguna Azul, T. terrestris también defeca semillas de $M$. flexuosa en lugares estacionalmente anegados, pero estas germinan y se establecen en esos sitios, sugiriendo que $T$. terrestris podría ser un eficiente dispersor de semillas de $M$. flexuosa, debido a los requerimientos de humedad para la fase de germinación y por el hábitat de las palmeras en áreas estacional o permanentemente inundadas.
Las diferencias estacionales en la remoción de frutos de palma real parecen estar relacionadas a la fenología de $M$. flexuosa. Una de las razones que podría explicar la variación del consumo de estos frutos para la época seca sería el cambio en el pico de fructificación de $M$. flexuosa y por lo tanto en la disponibilidad de frutos sobre el suelo, lo que ocasionaría que Dasyprocta cambie su área de forrajeo en tiempo y en espacio (Silvius 1999).

Nuestros resultados sugieren que C. paca dispersa las semillas de palma real a una distancia ligeramente mayor que $D$. punctata, aunque ambas especies alejan la mayoría de las semillas a distancias menores a $5 \mathrm{~m}$, muy cerca al promedio de distancia de dispersión de Astrocaryum aculeatissimum por Dasyprocta spp. reportado por Donatti et al. (2009). Las distancias de dispersión de semillas por D. punctata en la Laguna Azul son también similares a las reportadas para Attalea maripa (Fragoso 1994), B. excelsa (Peres et al. 1997) y Astrocaruym aculeatum (Jorge y Howe 2009) por Dasyprocta; pero son especialmente cercanas a las reportadas para Hymenaea courbaril, cuyas semillas, marcadas como en este estudio, fueron enterradas por Dasyprocta a un máximo de $12.1 \mathrm{~m}$ de distancia. Esto sugiere que las semillas dispersadas por Dasyprocta podrían resultar en poblaciones agregadas de las especies dispersadas. Sin embargo, Pires y Galetti (2012) reportan que D. leporina dispersa las semillas de Astrocaryum aculeatissimum a distancias máximas de $48.7 \mathrm{~m}$, con una media de $6.8 \mathrm{~m}$ y Prada-Villalobos y Araújo-Bagno (2012) reportan la exitosa dispersión de palma real por aves, especialmente Psittacidae, lo cual muestra que la dispersión de Mauritia podría no depender tanto de mamíferos como se había pensado originalmente, y que la dispersión por aves podría llevar las semillas mucho más lejos; pero esto no fue cuantificado en nuestro sitio de estudio. Dado que la palma real es la especie dominante en Laguna Azul, el alejamiento de las semillas desde los parentales no serviría como estrategia para evitar depredación, pues la mayoría de las semillas caen muy cerca a otra planta de la misma especie y la depredación probablemente no va a ser dependiente de la distancia a los parentales (Fricke et al. 2013).

Dasyprocta punctata lleva más semillas de $M$. flexuosa hacia el área inundable que C. paca. Dado que la germinación de $M$. flexuosa se ve favorecida en lugares inundables (Kahn et al. 1993), puede especularse que $D$. punctata sería un dispersor más efectivo que $C$. paca. Además, la presencia de agua representaría un medio de dispersión secundario para las semillas. Posiblemente la depredación por invertebrados es menor en semillas que quedan cubiertas por el agua, debido a que el agua interrumpiría la infestación de las semillas. Esto refuerza la idea que no es buena estrategia enterrar las semillas de $M$. flexuosa, por la frecuente inundación de las zonas donde se depositan las semillas. Sin embargo, esto dependerá de si la depredación de $M$. flexuosa por brúquidos ocurre antes o después de que las semillas caen de 
los árboles. Si los brúquidos ovipositan antes de la caída de los frutos, como en el caso de Astrocaryum standleyanum (Jansen et al. 2012), el efecto será insignificante.

La baja tasa de germinación de semillas de $M$. flexuosa podría deberse a la eliminación del mesocarpio y exocarpio por Dasyprocta, como fue reportado para A. maripa en la Isla Maraca, Brasil (Silvius y Fragoso 2002). La forma de remover la pulpa por los roedores, dejando al descubierto el endocarpio, facilitaría la oviposición de los brúquidos (Allen 1997; Silvius y Fragoso 2002).

\section{CONCLUSIONES}

Los frutos de Mauritia flexuosa son consumidos por D. punctata, C. paca, T. terrestris, M. americana y roedores pequeños en la Reserva Pilón Lajas; pero la mayor remoción se da por $D$. punctata y C. paca. Ambas especies lleva las semillas a distancias de aproximadamente $14 \mathrm{~m}$, pero la mayoría son depositadas a distancias $<5 \mathrm{~m}$. Cuniculus paca dispersa las semillas de M. flexuosa a mayor distancia que D. punctata, pero lleva las semillas principalmente en dirección al área no inundable, que podría ser un sitio menos seguro para germinación y establecimiento. Una proporción mínima de las semillas dispersadas por ambas especies llega a germinar. La mayoría se pudren o son depredadas. Las semillas tendrían mayor probabilidad de sobrevivir si se las entierra, que si se depositan sobre el suelo; y la probabilidad de una semilla de ser depredada es mayor en la época seca. En este estudio no se obtuvo evidencias de que $D$. punctata entierre las semillas de $M$. flexuosa y éste parece ser el primer reporte de ausencia del comportamiento de scatterhoarding para Dasyprocta para semillas grandes; este hecho podría ampliar la plasticidad del comportamiento de esta especie de acopiar semillas, ante diferencias en abundancia de esos recursos.

\section{AGRADECIMIENTOS}

Walfrido Tomas (Empresa Brasileira de Pesquisa Agropecuária - Embrapa) nos enseñó a marcar las semillas con metal. Enzo Aliaga, Ramiro Bustamante, M. Fleury, Narel Paniagua, Nathaniel Wheelwright y dos revisores anónimos comentaron versiones preliminares del manuscrito. Agradecemos el financiamiento del Instituto de Ecología de la Universidad Mayor de San Andrés y la ONG BIOTA. Mauricio Ocampo preparó la figura 1.

\section{BIBLIOGRAFÍA CITADA}

Alho, C. 1982. Brazilian rodents: their habitats and habits. In: Mares M.; Genoways, H. (Ed.) Mammalian Biology in South America. Pittsburgh University, Pittsburgh, p.143-158

Aliaga-Rossel, E; Kays, R; Fragoso, J. 2008. Home-range use by the central American agouti (Dasyprocta punctata) on Barro Colorado Island Panama. Journal of Tropical Ecology, 24:367 - 374.
Allen, C. 1997. Frugivores, palms, and conservation in Amazonia. Master of Arts Thesis. University of Florida, Gainesville. 94p.

Andreazzi, C.S.; Pires, A.S.; Fernandez, F.A. 2009. Mamíferos e palmeiras neotropicais: interaçôes em paisagens fragmentadas. Oecologia Brasiliensis, 13: 554-574.

Bodmer, R. 1991. Strategies of seed dispersal and seed predation in amazonian ungulates. Biotropica, 23: 255-261.

Bradford, D.; Smith, C. 1977. Seed predation and seed number in Scheelea palm fruits. Ecology, 58:667-673.

Braun, A. 1988. El cultivo de las palmas en el trópico. Cervantes, Caracas, $67 \mathrm{p}$.

Donatti, C.I.; Guimaraes Jr, P.R.; Galetti, M. 2009. Seed dispersal and predation in the endemic Atlantic rainforest palm Astrocaryum aculeatissimum across a gradient of seed disperser abundance. Ecological Research, 24: 1187-1195.

Emsens, W.J.; Suselbeek, L.; Hirsch, B.T.; Kays, R.; Winkelhagen, A.J.; Jansen, P A. 2013. Effects of food availability on space and refuge use by a neotropical scatterhoarding rodent. Biotropica, 45: 88-93.

Forget, P.M.; Milleron, T. 1991. Evidence for secondary seed dispersal by rodents in Panama. Oecología. 87: 596-599.

Fragoso, J. M. 1994. Large mammals and the community dynamics of an amazonian rain forest. Dissertation of Doctor of Philosophy. University of Florida, Gainesville. 210 p.

Fragoso, J.M. 1997. Desapariciones locales del baquiro labiado (Tayassu pecari) en la Amazonia: Migración, sobre-cosecha, o epidemia? In: Fang, T.; Bodmer, R.; Aquino, R; Valqui, M. (Ed.). Manejo de Fauna Silvestre en la Amazonía. Instituto de Ecología. La Paz. p.309-312.

Fragoso, J.; Huffman, J. 2000. Seed-dispersal and seedling recruitment patterns by the last Neotropical megafaunal element in Amazonia, the tapir. Journal of Tropical Ecology, 16:369-385.

Fricke, E.C.; Simon, M.C.: Reagan, K.M.; Levey, D.J.; Riffell, J.A.; Carlo, T.A.; Tewksbury, J.J. 2013. When condition trumps location: seed consumption by fruit-eating birds removes pathogens and predator attractants. Ecology Letters, 16:1031-1036.

Galetti, M.; Donatti, C.I.; Steffler, C.; Genini, J.; Bovendorp, R. S.; Fleury, M. 2010. The role of seed mass on the caching decision by agoutis, Dasyprocta leporina (Rodentia: Agoutidae). Zoologia, 27: 472-476.

Gálvez, D.; Kranstauber, B.; Kays, R.W.; Jansen, P.A. 2009. Scatter hoarding by the Central American agouti: a test of optimal cache spacing theory. Animal Behaviour, 78: 1327-1333.

Hirsch, B.T.; Kays, R.; Jansen, P.A. 2012a. A telemetric thread tag for tracking seed dispersal by scatter-hoarding rodents. Plant Ecology, 213: 933 - 943.

Hirsch, B. T.; Kays, R.; Pereira, V. E.; Jansen, P. A. 2012b. Directed seed dispersal towards areas with low conspecific tree density by a scatter-hoarding rodent. Ecology letters, 15: 1423-1429.

Holm, J.A.; Miller, C.J.; Cropper Jr., W.P. 2008. Population dynamics of the dioecious amazonian palm Mauritia flexuosa: Simulation analysis of sustainable harvesting. Biotropica, 40: 550-558. 
Howe, H.F.; Miriti, M.N. 2004. When seed dispersal matters. BioSience, 54: 651-660.

Ibisch, P.L.; Beck, S.G.; Gerkmann, B.; Carretero, A. 2003. Ecoregiones y ecosistemas. In: Ibisch, P.L.; Mérida, G.; (Ed.). Biodiversidad: la riqueza de Bolivia: estado de conocimiento y conservación. Editorial FAN. Santa Cruz, Bolivia p.47-88.

Jansen, P.A.; Bongers, F.; Hemerik, L. 2004. Seed mass and mast seeding enhance dispersal by a neotropical scatter-hoarding rodent. Ecological Monographs, 74: 569-589.

Jansen, P.A.; Hirsch, B.T.; Emsens, W.J.; Zamora-Gutierrez, V.; Wikelski, M.; Kays, R. 2012. Thieving rodents as substitute dispersers of megafaunal seeds. Proceedings of the National Academy of Sciences, 109: 12610-12615.

Johansson, B. 2009. Stay below water!-a strategy to avoid seed predators:-seed survival and germination of Mauritia flexuosa in southeastern Peru. Masters thesis. Linköpings Universitet, Suecia. 34p.

Jorge, M.L.S.; Howe, H.F. 2009. Can forest fragmentation disrupt a conditional mutualism? A case from central Amazon. Oecologia, 161: 709-718.

Kahn, F.; Mejía, K.; Moussa, F.; Gómez, D. 1993. Mauritia flexuosa, la más acuática de las palmeras amazónicas. In: Kahn, F.; León, B.; Young, K.R. (Ed.). Las plantas vasculares en las aguas continentales del Perú. IFEA. Lima, p.287-321.

Kiltie, R. 1981. Distribution of palm fruits on a rain forest floor: Why white-lipped peccaries forage near objects. 1981. Biotropica, 13: 141-145.

Lopes, A.; Albuquerque, J.; Silva, M.; Shrimpton, R. 1980. Aspectos nutritivos de algunos frutos da Amazonía. Acta Amazonica, 10:755-758.

Moraes, M. 1989. Ecología y formas de vida de las palmas bolivianas. Ecología en Bolivia, 13: 33-45.

Moraes, M. 1993. Palmae. In: Killeen, T.; García, E.; Beck, S. (Ed.). Guía de Árboles de Bolivia. Instituto de Ecología, La Paz. p.612-628.

Morris, D. 1962. The behaviour of the green acouchis (Myoprocta pratti) with special reference to scatter hoarding. Zoological Society, 139: 701-733.

Peres, C.; Schiesari, K.; Dias-Leme, C. 1997. Vertebrate predation of Brazil nuts (Bertholletia excelsa, Lecythidaceae), an Agouti dispersed Amazonian seed crop: a test of the escape hypotesis. Journal of tropical Ecology, 13: 69-79.

Pires, A.S.; Galetti, M. 2012. The agouti Dasyprocta leporina (Rodentia: Dasyproctidae) as seed disperser of the palm Astrocaryum aculeatissimum. Mastozoología Neotropical, 19: 147-153.

Prada-Villalobos, M; Araújo-Bagno, M. 2012. Avian frugivores feeding on Mauritia flexuosa (Arecaceae) fruits in Central Brazil. Revista Brasileira de Ornitologia, 20: 26-29.

Quiroga, D.; Roldán, A.I. 2001. The fate of Attalea phalerata seeds dispersed to a tapir latrine. Biotropica, 33: 472-477.

Ríos, R.; Pacheco, L.F. 2006. The effect of dung on post-dispersal seed predation of Attalea phalerata (Arecaceae) by bruchid beetles. Biotropica, 38: 778-781.
Rojas-Robles, R.; Stiles, G.F.; Muñoz-Saba, Y. 2012. Frugivoría y dispersión de semillas de la palma Oenocarpus bataua (Arecaceae) en un bosque de los Andes colombianos. Revista de Biología Tropical, 60: 1445-1461.

Roldán, A.I; Simonetti, J.A. 2001. Plant-Mammal Interactions in Tropical Bolivian Forests with Different Hunting Pressures. Conservation Biology, 15: 617-623.

Siegel, S.; Castellan, J. 1995. Estadística no paramétrica aplicada a las ciencias de la conducta. Trillas. México, 437p.

Silvius, K.M. 1999. Interactions among Attalea palms, bruchid beetles, and neotropical terrestrial fruit-eating mammals: implications for the evolution of frugivory. Dissertation of Doctor of Philosophy. University of Florida, Gainesville. 202p.

Silvius, K.M.; Fragoso, J.M.V. 2002. Pulp handling by vertebrate seed dispersers increases palm seed predation by bruchid beetles in the northern Amazon. Journal of Ecology, 90: 1024-1032.

Silvius, K.M.; Fragoso, J.M.V. 2003. Red-rumped Agouti (Dasyprocta leporina) home range use in an Amazonian forest: Implications for the aggregated distribution of forest trees. Biotropica, 35:74-83.

Simonetti, J.A.; Huareco, I. 1999. Uso de huellas para estimar diversidad y abundancia relativa de los mamíferos de la reserva de la Biosfera-Estación Biológia del Beni, Bolivia. Mastozoología Neotropical, 6:139-144.

Smythe, N. 1970. Relationships between fruiting seasons and seed dispersal methods in a tropical forest. American Naturalist, 104:25-35.

Smythe, N. 1978. The Natural History of the Central American Agouti (Dasyprocta punctata). Smithsonian Contributions to Zoology, 257: 1-60.

Smythe, N.; Glanz, W.; Leigh, E. 1982. Regulación de la población de algunos frugívoros terrestres. In: Leigh, E.; Rand, A.; Windsor, D. (Ed.) Ecología de un Bosque Tropical, Ciclos Estacionales y Cambios a Largo Plazo. Smithsonian Institution, Colombia. p.305-315.

Soares, S.; Ribeiro, M. 1995. Evidências de dispersão de sementes mediada por mamíferos caviomorfos em Hymenaea oblongifolia. Botánica, 11:265-273.

Tuck-Haugaasen, J.M.; Haugaasen, T. Peres, C.A.; Gribel, R.; Wegge, P. 2012. Fruit Removal and Natural Seed Dispersal of the Brazil Nut Tree (Bertholletia excelsa) in Central Amazonia, Brazil. Biotropica, 44: 205-210.

Visser, M.D.; Muller-Landau, H.C.; Wright, S J.; Rutten, G.; Jansen, P.A. 2011. Tri-trophic interactions affect density dependence of seed fate in a tropical forest palm. Ecology letters, 14: 1093-1100.

Wang, B.C.; Smith, T.B. 2002. Closing the seed dispersal loop. Trends in Ecology \& Evolution, 17: 379-386.

Zar, J.H. 1999. Biostatistical Analysis (4a edición). Prentice Hall International, Inc. New Jersey, 663p.

Recebido em 23/01/2014

Aceito em 03/07/2014 
